\title{
Watering and nitrogen and potassium fertilization: the significance of abiotic control on Gynura procumbens (Lour.) Merr herbs in Malaysia for better growth and secondary metabolite enrichment
}

\begin{abstract}
Environmental changes have led to cellular adjustment and adaptation in plant growth. External factors have, for example, influenced the growth pattern of Gynura procumbens plants and led to production of specific secondary metabolite internally for the purpose of differentiation and conditional interaction. These developmental patterns and production of metabolites are expressional characteristics of the plant, and so growers can have only a restricted range of movement or limited control over their reaction to environmental changes compared to their reaction to human or animal interactions. Even though metabolite production is pervasive among the plants, the need to explore abiotic control strategies for regulating the patterns of growth of Gynura procumbens as well as their accumulation of metabolites has been shown to be significant in recent studies of plant-abiotic interactions.
\end{abstract}

Keyword: Abiotic; Growth; Gynura procumbens; Herbs; Metabolite 